\title{
Eficacia de la combinación de Abamectina-Derquantel y de la Moxidectina en cabras lecheras naturalmente parasitadas con nematodos gastrointestinales en el Valle de Lerma, Argentina
}

\author{
Olmos $\mathrm{LH}^{1,2}$, Martínez $\mathrm{GM}^{3}$, Alfaro $\mathrm{RJ}^{3}$, Diaz $\mathrm{JP}^{4}$, Suarez $\mathrm{VH}^{3}$
}

1. Instituto Nacional de Tecnología Agropecuaria (INTA), -CIAP-Instituto de Investigación Animal del Chaco Semiárido -Área de Investigación en Salud Animal, Estación Experimental Agropecuaria (EEA) Salta (Cerrillos), Argentina.

2. Facultad de Ciencias Agrarias y Veterinarias, Universidad Católica de Salta, Argentina.

3. Estación Experimental Agropecuaria Salta (EEA Salta), INTA, Argentina.

4. Facultad de Ciencias Naturales, Universidad Nacional de Salta, Argentina.

* Correspondencia: Leandro Olmos, INTA EEA Salta. Ruta Nacional 68 km 172, 4403 Cerrillos, Salta, Argentina. E-mail: olmos.leandro@inta.gob.ar

Recibido: 23 Abril 2021. Aceptado: 4 Agosto 2021. Disponible en línea: 16 Diciembre 2021

Editor: P. Beldomenico

\begin{abstract}
RESUMEN. El impacto productivo de los nematodos gastrointestinales (NGI) sobre los de pequeños rumiantes es conocido a nivel mundial. Las infestaciones por NGI constituyen una limitante de alto impacto en la producción de rumiantes y las perdidas comprendidas por este problema sanitario varían desde reducciones subclínicas en la ganancia de peso hasta la muerte de animales con altas cargas parasitarias. El objetivo de este trabajo es presentar los resultados de un test de eficacia a campo utilizando una combinación comercial de Abamectina + Derquantel y del Moxidectin en cabras lecheras naturalmente infestadas con NGI en el Valle de Lerma, provincia de Salta. El ensayo se llevó a cabo en la Unidad Tambo Caprino de la Estación Experimental Agropecuaria Salta del INTA. Para la evaluación de eficacia de la droga en estudio, se realizó el test de reducción de conteos de huevos. En los coprocultivos pre-tratamientos se pudo observar una gran prevalencia de Trichostrongylus spp. (71\%). seguido de Teladorsagia spp. (21\%) y Haemonchus spp. (8\%). En cuanto a los resultados del TRCH, en el GDQ se observaron valores del $100 \%$ de PRCH, mientras que en el GMX se observó un PRCH de 95,5\%, donde la eficacia para Haemonchus spp. y Teladorsagia spp. fue del $100 \%$ y para Trichostrongylus spp. del $81,1 \%$.Los resultados observados en este trabajo permiten concluir que en las condiciones de campo en donde fue llevado a cabo el TRCH, la combinación Derquantel-Abamectina posee una alta eficacia contra los géneros presentes.
\end{abstract}

SUMMARY. Efficacy of combining Abamectin-Derquantel and Moxidectin in dairy goats naturally infected by gastrointstinal nematodes in Valle de Lerma, Argentina. The productive impact of gastrointestinal nematodes (NGI) of small ruminants are known worldwide. Infestations by NGI constitute a limiting factor in the production of ruminant and the losses included by this sanitary problem vary from subclinical reductions in weight gain to the death of animals with high parasite loads. The objective of this work is to present the results of an efficacy trial using a commercial combination of Abamectin + Derquantel and Moxidectin in dairy goats naturally infested with NGI in the Lerma Valley, Salta province. The test was carried out in the Dairy Goat Unit of the Salta Agricultural Experiment Station of INTA. In the pretreatment stool cultures, a high prevalence of Trichostrongylus spp. (71\%). followed by Teladorsagia spp. (21\%) and Haemonchus spp. (8\%) was observed. Regarding the results of the TRCH, in the GDQ values of $100 \%$ of PRCH were observed, while in the GMX a PRCH of $95.5 \%$ is shown, where the efficacy for Haemonchus spp. and Teladorsagia spp. was $100 \%$ and for Trichostrongylus spp. $81.1 \%$. The results observed in this work allow us to conclude that under the field conditions where TRCH was carried out, the Derquantel-Abamectin combination has high efficacy against the genera present.

Palabras clave: Eficacia, Derquantel, Abamectina, Nematodes, Cabras

Keywords: Efficacy, Derquantel, Abamectin, Nematodes, Goats

\section{Introducción}

El impacto productivo de los nematodos gastrointestinales (NGI) sobre los de pequeños rumiantes son conocidos a nivel mundial (Suarez et al., 2013). Las infestaciones por NGI constituyen una limitante de alto impacto en la producción de rumiantes y las perdidas comprendidas por este problema sanitario varían desde reducciones subclínicas en la ganancia de peso hasta la muerte de animales con altas cargas parasitarias (Suarez et al, 1990; Anziani y Fiel, 2015). Dicho impacto productivo en explotaciones caprinas esta descripto en algunos trabajos a nivel nacional tanto en pérdidas de carne como de leche. 
En la provincia de San Luis se estableció una pérdida de entre 4 y $6 \mathrm{Kg}$ al comparar el peso entre cabras infestadas naturalmente con $\mathrm{NGI}$ con y sin tratamiento antihelmíntico (Rossanigo y Frigerio, 2000), mientras que en la provincia de Salta un estudio con cabras lecheras en ordeñe arrojo un aumento del $41,8 \%$ en el rinde lácteo total a favor de un grupo de cabras tratadas al compararlas con un grupo sin tratamiento naturalmente infestadas (Suarez et al., 2017).

Actualmente, la herramienta más difundida en el control de $\mathrm{NGI}$ es mediante la aplicación de antihelmínticos (Cerutti et al., 2018). Sin embargo, la aparición del fenómeno de resistencia antihelmíntica (RA) constituye un riesgo importante en la producción de rumiantes en sistemas pastoriles al aumentar los costos relacionados con el control de los NGI (Anziani y Fiel, 2015). En el NOA existen algunos reportes en donde se describe la presencia de RA antihelmíntica al albendazol y de la moxidectina en majadas caprinas (Aguirre et al., 2000, Suarez et al., 2012) y a la abamectina (Aguirre et al., 2002).

En este contexto la búsqueda de alternativas terapéuticas en base a nuevas moléculas o combinaciones de estas con antihelmínticos de amplio espectro ya conocidos, pueden ser herramientas que ayuden a formular planes de control estratégicos con el fin de disminuir el impacto de los NGI en las explotaciones pecuarias. Una de las drogas antihelmínticas últimamente desarrolladas fue el Derquantel (2-deoxyparaherquamide), derivado semisintético de la clase de los spiroindoles, el cual basa su efecto antihelmíntico en un antagonismo nicotínico (Woods et al., 2012). Comercialmente, este producto solo está disponible en combinación con Abamectina con el fin de aumentar su espectro de acción y demorar la aparición del de resistencia antihelmíntica (Cerutti et al., 2018).

Por lo tanto, el objetivo de este trabajo es el de dar a conocer los resultados de un test de eficacia a campo utilizando una combinación comercial de Abamectina + Derquantel (ABA-DQT) y de la Moxidectina (MXD) en cabras lecheras naturalmente infestadas con NGI en el Valle de Lerma, provincia de Salta.

\section{Materiales y métodos}

El ensayo se llevó a cabo en la Unidad Tambo Caprino de la Estación Experimental Agropecuaria Salta del INTA, Cerrillos provincia de Salta $\left(24^{\circ} 53^{\prime} 32\right.$. $\left.65^{\circ} 28^{\prime} 26.4^{\prime \prime} \mathrm{W}\right)$. Esta se halla ubicada en el Valle de Lerma, ecorregión caracterizada por un clima subandino serrano (altitud de $1050 \mathrm{msnm}$ ) con un régimen de lluvias estivales de diciembre a abril, seguido por un período de sequía invierno-primaveral que se prolonga de mayo a noviembre (Bianchi y Bravo, 2008).

Para la evaluación de eficacia de la droga en estudio, se realizó el test de reducción de conteos de huevos
(TRCH). Dicho test determina la eficacia de una droga basándose en la reducción de los huevos por gramo de materia fecal (Hpg) unos 10-15 días posteriores a la aplicación de alguna droga antihelmíntica. Como animales experimentales, se utilizaron cabras preñadas y cabrillas de raza Saanen, seleccionadas previamente en base al Hpg, el cual debía ser mayor a 200 y mantenidos sobre una pastura de alfalfa con una suplementación en base a maíz.

Los animales fueron divididos en 3 grupos de 15 animales cada uno: un grupo control (GCT) sin desparasitar, un grupo tratado con Moxidectina al $1 \%$ (GMX) a razón de $1 \mathrm{ml}$ por cada $30 \mathrm{Kg}$ PV por vía subcutánea y otro desparasitado con la combinación Abamectina $0,1 \%$ Derquantel $1 \%$ (GDQ) a razón de $1,2 \mathrm{ml}$ cada $5 \mathrm{Kg}$ PV por vía oral. La dosificación de las drogas fue establecida tomando en cuenta las diferencias metabólicas con los ovinos (principalmente a nivel hepático) de la especie caprina considerando una dosis $20 \%$ mayor $(1,2$ $\mathrm{ml} / 5 \mathrm{~kg} \mathrm{PV}$ ) a la prescripta por el fabricante para la especie ovina en el caso de la combinación ABA-DQT y del $50 \%$ mayor en el caso del moxidectin $(1 \mathrm{ml} / 30 \mathrm{~kg}$ $\mathrm{PV})$. Los muestreos de materia fecal fueron realizados al momento de la desparasitación y 14 días posteriores a la realización de dicho tratamiento.

Las muestras obtenidas fueron procesadas mediante la técnica de McMaster modificada por Roberts y 0 'Sullivan, (1949) para obtener el conteo de Hpg y para la determinación de los géneros parasitarios se realizaron coprocultivos en cada uno de los grupos experimentales. Para esto se obtuvieron alícuotas de aproximadamente $2 \mathrm{~g}$ por muestra, las cuales fueron incubadas en estufa y las larvas desarrolladas fueron recolectadas 10 días post-incubación mediante la técnica propuesta por Suárez (1997) y posteriormente identificadas según las claves morfológicas descriptas por Niec, (1968).

La eficacia de las drogas en estudio esta expresada como porcentaje de reducción de los conteos de huevos (PRCH) y fue calculado empleando la fórmula propuesta por Dash (1988) a partir de la fórmula de Abbott (mod. Henderson y Tilton, 1955): PRCH 100 (1$[\mathrm{T} 2 / \mathrm{T} 1] \times[\mathrm{C} 1 / \mathrm{C} 2])$, donde: $\mathrm{C} 1=$ promedio de $\mathrm{Hpg}$ del grupo control pre-tratamiento día $0 . \mathrm{C} 2=$ promedio de Hpg del grupo control pos-tratamiento día 13. T1= promedio de $\mathrm{Hpg}$ del grupo tratado pre-tratamiento día 0 . $\mathrm{T} 2=$ promedio de $\mathrm{Hpg}$ del grupo tratado postratamiento día 14. Según esto los resultados de eficacia son presentados en porcentaje. A su vez, en el caso de la eficacia calculada por género parasitario, se determinó el valor de $\mathrm{Hpg}$ en base a la prevalencia observada en los coprocultivos. Se consideró como eficaz a un valor PRCH mayor al $95 \%$.

En lo referente al estatus de resistencia antihelmíntica de la población de nematodes gastrointestinales presente en las pasturas donde se realizó el ensayo, solo existe una cita sobre la pobre eficacia del moxidectin sobre Trichostrongylus spp y de la eficacia del 
monepantel tanto sobre este género como contra Haemonchus y Teladorsagia (Suarez et al., 2012).

\section{Resultados}

En los coprocultivos pre-tratamientos se pudo observar una gran prevalencia de Trichostrongylus spp. (71\%), seguido de Teladorsagia spp. (21\%) y Haemonchus spp. (8\%). En cuanto a los resultados del TRCH, en el GDQ se observaron valores del $100 \%$ de $\mathrm{PRCH}$, mientras que en el GMX se observó un PRCH de 95,5\%, donde la eficacia para Haemonchus spp. y Teladorsagia spp. fue del $100 \%$ y para Trichostrongylus spp. del $81,1 \%$. Estos resultados se resumen en las Tablas 1 y 2.
Tabla 1. Valores de Hpg pre y post tratamiento y porcentaje de reducción del conteo de huevos (PRCH) general por tratamiento antiparasitario. (GCT: Grupo control; GMX: Grupo tratado con moxidectin; GDQ: Grupo tratado con la combinación Abamectina-Derquantel).

\begin{tabular}{|c|c|c|c|}
\hline Grupo & $\begin{array}{c}\text { Hpg } \\
\text { pre-tratamiento }\end{array}$ & $\begin{array}{c}\text { Hpg } \\
\text { post-tratamiento }\end{array}$ & PRCH (\%) \\
\hline GCT & 484,7 & 667,9 & - \\
\hline GMX & 432 & 21,4 & $95,5 \%$ \\
\hline GDQ & 520 & 0 & $100 \%$ \\
\hline
\end{tabular}

Tabla 2. Prevalencia por géneros pre tratamiento y porcentaje de reducción del conteo de huevos (PRCH) por géneros por tratamiento antiparasitario. (GCT: Grupo control; GMX: Grupo tratado con Moxidectina; GDQ: Grupo tratado con la combinación Abamectina-Derquantel).

\begin{tabular}{|c|c|c|c|c|c|c|}
\hline Grupo & $\begin{array}{c}\text { Trichostrongylus } \\
\text { Pre-trat. }\end{array}$ & $\begin{array}{c}\text { Haemonchus } \\
\text { Pre-trat. }\end{array}$ & $\begin{array}{c}\text { Teladorsagia } \\
\text { Pre-trat. }\end{array}$ & $\begin{array}{c}\text { PRCH (\%) } \\
\text { Trichostrongylus }\end{array}$ & $\begin{array}{c}\text { PRCH (\%) } \\
\text { Haemonchus } \\
\text { Teladorsagia }\end{array}$ \\
\hline GCT & $71 \%$ & $21 \%$ & $8 \%$ & - & - \\
\hline GMX & $60 \%$ & $20 \%$ & $20 \%$ & $81,1 \%$ & $100 \%$ & $100 \%$ \\
\hline GDQ & $80 \%$ & $10 \%$ & $10 \%$ & $100 \%$ & $100 \%$ & $100 \%$ \\
\hline
\end{tabular}

\section{Discusión}

La combinación de antihelmínticos con diferentes características farmacológicas, puede ser sugerida como una opción en el tratamiento de nematodos gastrointestinales con el fin de controlar poblaciones parasitarias con resistencia mixta o demorar la aparición del fenómeno de resistencia antihelmíntica (Geary et al., 2013; Anziani y Fiel, 2015). Por otro lado, algunos trabajos demuestran que en contextos en donde la RA se encuentra en altos niveles, estas combinaciones podrían no ser de tanta utilidad (Entrocasso et al., 2008; Anziani y Muchiut, 2014). En cuanto a esto, la información disponible sobre las interacciones que se puedan observar entre los antihelmínticos aún no está del todo esclarecida (Anziani y Fiel, 2015). Por lo tanto, los trabajos realizados con este tipo de combinaciones permiten aportar al conocimiento de dichas interacciones.

En la especie caprina, la utilización de antihelmínticos merece una consideración especial debido a que existe un espectro muy reducido de drogas registradas para esta especie (Anziani y Fiel, 2015). Tal es el caso de la combinación ABA-DQT, la cual no contempla su utilización en los caprinos. Sin embargo, a partir de los resultados obtenidos en el presente trabajo, se pudo observar que este producto puede ser empleado en dicha especie con una eficacia antihelmíntica óptima. Es de destacar, que los animales utilizados se encontraban cursando el último tercio de la gestación no mostraron ningún efecto adverso sobre su progenie (aborto, malformaciones congénitas, nacimiento de cabritos débiles), por lo cual si bien el número de animales es poco como para sacar conclusiones, no deja de ser una observación de relevancia.

En las condiciones de campo en donde fue llevado a cabo el TRCH, la combinación ABA-DQT posee una alta eficacia contra los géneros presentes. En forma general, estos resultados coinciden con los observados por Cerutti et al., (2018) en caprinos, los cuales observaron un $\mathrm{PRCH}$ del $100 \%$. Sin embargo, es de destacar que los resultados obtenidos por dichos autores fueron realizados en un contexto de alta prevalencia de Haemonchus contortus, mientras que lo determinado en el presente estudio demuestra a su vez una gran eficacia contra el género Trichostrongylus spp. Por otra parte, en el caso de la MXD, se observó alta eficacia sobre los géneros Teladorsagia y Haemonchus y una eficacia disminuida $(84,8 \%)$ sobre el género Trichostrongylus.

Cabe destacar que al tratarse de una formulación combinada entre un spiroindol y una lactona macrocíclica no es posible separar la eficacia de cada droga. Sin embargo, en el caso de Trichostrongylus spp., tomando en cuenta que la eficacia del MXD resultó se baja coincidiendo con observaciones previas en esta misma población (Suarez et al., 2012), que al tratarse también la ABM de una lactona macrocíclica la eficacia de la combinación fue debida mayormente al Derquantel. Por otro lado, para los casos del género Haemonchus y Teladorsagia, al no haber podido 
conformar grupos tratados con solo una de las drogas, es imposible inferir que droga tuvo más participación sobre la alta eficacia observada.

Los resultados observados en este trabajo, coinciden con lo observado en otros estudios en donde la combinación ABA-DQT demostró una eficacia optima contra géneros como Trichostrongylus (Kaminsky et al., 2011), Teladorsagia (Geurden et al., 2012) y Haemonchus (Cerutti et al., 2018). Es importante mencionar que el espectro del DQT por si solo es considerado reducido. Dicho espectro está determinado por una alta eficacia contra estadios adultos de H. contortus, Trichostrongylus spp. y Teladorsagia spp, mostrando una baja eficacia contra estadios inmaduros (L4) de H. contortus (Kaminsky et al., 2011). Por lo tanto, si bien es una molécula que puede ser tenida en cuenta para ser usada en un contexto de resistencia, en el caso de $H$. contortus se debe tener en cuenta la posibilidad de reinfecciones rápidas por la sobrevivencia y posterior desarrollo de las L4 que no estarían afectadas por el tratamiento. Por otro lado, los resultados observados en el presente trabajo, ponen a la combinación ABA-DQT como una alternativa terapéutica optima en el caso de un contexto de resistencia con alta prevalencia de Trichostrongylus spp.

En función de los resultados obtenidos es posible concluir que la combinación ABA-DQT es una formulación que puede ser utilizada en caprinos y que puede ser una alternativa factible de ser considerada en contextos de resistencia antihelmíntica. Por otro lado, es importante destacar esta estrategia de control debe ser monitoreada con el uso del TRCH y debe ser utilizada en base al monitoreo previo de la carga parasitaria mediante el conteo de $\mathrm{Hpg}$ y/o en base a criterios de tratamientos selectivos como la escala de FAMACHA con el fin de demorar la aparición del fenómeno de resistencia antihelmíntica y poder realizar un control sustentable de los nematodos gastrointestinales en las majadas caprinas.

\section{Agradecimientos}

Queremos agradecer al Med Vet Claudio Gauna de la empresa Zoetis por el aporte del antiparasitario Startec (Abamectina 0,1\%+Derquantel 1\%), el cual nos permitió llevar a cabo el presente trabajo de investigación.

\section{Referencias}

Aguirre DH, Cafrune MM, Viñabal AE, Salatin AO. 2000 Mortalidad por nematodiasis asociada a la ineficacia del albendazole en cabras lecheras del valle de Lerma (Salta). Rev. Arg. Prod. Anim. 20: 341-343.

Aguirre DH, Cafrune MM, Viñabal AE, Salatin AO. 2002. Resistencia a las avermectinas en Trichostrongylus colubriformis de cabras del noroeste argentino. Vet. Arg. 19: 489-496.
Anziani, OS, Muchiut S. 2014. Resistencia antihelmíntica múltiple (closantel, febendazole, ivermectina y levamisole) en Haemonchus spp. parasitando a ovinos en la provincia de Santa Fe. Ineficacia de una triple combinación de estas drogas para su control. Rev. Med. Vet. (Buenos Aires) 95:22-27.

Anziani OS, Fiel CA. 2015. Resistencia a los antihelmínticos en nematodos que parasitan a los rumiantes en la Argentina. Rev. Invest. Agrop. 41: 34-46.

Bianchi AR, Bravo GC. 2008. Ecorregiones Norandina: Descripción, subregiones, agroecosistemas, sistemas productivos y cartografía regional Ed. INTA, EEA Salta. 60 pp.

Cerutti J, Cooper LG, Torrents J, Suarez Archilla GA, Anziani, OS. 2018. Eficacia reducida de derquantel y abamectina en ovinos y caprinos con Haemonchus sp resistentes a lactonas macrocíclicas. Eficacia reducida de derquantel y abamectina en ovinos y caprinos con Haemonchus sp resistentes a lactonas macrocíclicas. Rev. Vet. 29: 22-25

Dash KM, Hall E, Barger IA. 1988. The role of arithmetic and geometric mean worm egg counts in faecal egg count reduction tests and in monitoring strategic drenching programs in sheep. Aust. Vet. J. 65: 66-68.

Entrocasso C, Álvarez L, Mananzza J. Lifcschitz A, Borda B, Virkel G, Mottier L, Lanusse C. 2008. Clinical efficacy assessment of the albendazole-ivermectina combination in lambs parasitized with resistant nematodes. Vet. Parasitol. 155: 249-256.

Geary TG, Hosking BC, Skuce PJ, von Samsom Himmelstjern AG, Maeden S, Holdsworth P, Pomroy W, Vercruysse J. 2013. World Association for the Advancement of Veterinary Parasitology (W.A.A.V.P.) Guideline: Anthelmintic combination products targeting nematode infections of ruminants and horses. Vet. Parasitol. 190: 306-316.

Geurden T, Hodge A, Winstanley D, Bartley D, Taylor M, Morgan C, Fraser S, Maeder S, Bartram D. 2012. The efficacy of a combined oral formulation of derquantel-abamectin against anthelmintic resistant gastro-intestinal nematodes of sheep in the UK. Vet Parasitol 189: 308-316.

Henderson CF, Tilton EW. 1955. Tests with acaricides against the brown wheat mite. J. Econ. Ent. 48: 157-161.

Kaminsky R, Bapst B, Stein PA, Strehlau GA, Allan BA, Hosking $B C$, Rolfe PF, Sager H. 2011. Differences in efficacy of monepantel, derquantel and abamectin against multi-resistant nematodes of sheep. Parasitol. Res. 109: 19-23.

Roberts F, O'Sullivan P. 1949. Methods for egg counts and larval culture for strongyles infesting gastrointestinal tract of cattle. Aust. J. Agric. Res. 1: 99-102.

Rossanigo C, Frigerio K. 2000. Epidemiology and effects of nematode infections on the production of Criolla goats. Proceedings $7^{\circ}$ International Conference on Goats 2: 802-805.

Suarez VH, Larrea S, Busetti MR, Bedotti DO, Bulman GM, Ambrustolo RR. 1990. Nematodes gastrointestinales ovinos: Su control y efecto sobre los parámetros epizootiológicos, hematológicos y productivos en la Región Semiárida Pampeana (Argentina). Therios 15: 156-173. 
Suarez VH, Fondraz M, Viñabal A, Martínez GM, Salatin AO. 2013. Epidemiología de los nematodes gastrointestinales en caprinos lecheros en los valles templados del NOA, Argentina. Rev. Invest. Agrop. 39: 191-197.

Suarez VH. 1997. Diagnóstico de las parasitosis internas de los rumiantes en la región de invernada. Técnicas e Interpretación. Boletín de Divulgación Técnica (INTA-Anguil) 56: 22pp.

Suarez VH., Almudevar F, Fondraz M, Viñabal A. 2012. Eficacia del monepantel en caprinos lecheros en los valles templados del Noroeste Argentino. En: Programa de Ámbito Nacional Leche. Producción técnica-científica de Proyecto Cartera 20062009 / 2010-2012. (Eds, Taverna M., Comeron, E.A., Suarez, V.H.) Producciones INTA, Argentina 9: 885 pp. ISBN 878987679180.

Suarez VH, Martínez GM, Viñabal AE, Alfaro JR. 2017. Epidemiology and effect of gastrointestinal nematodes on dairy goats in Argentina. Onder. J. Vet. Res. 84: 1240.

Woods DJ, Maeder SJ, Robertson AP, Martin, RJ, Geary TG, Thompson DP, Conder GA. 2012. Discovery, mode of action, and commercialization of derquantel. Paras. Helminth. Targets, Screens, Drugs Vac. 297-307 pp. 